\title{
Construire des logements adaptés aux personnes âgées: une analyse par les territoires de vie à Lyon (France)
}

\author{
Pierre-Marie Chapon, Lyon et Paris, Florent Renard, \\ Lyon
}

\section{Introduction}

La France devrait compter 22,3 millions de personnes âgées de 60 ans et plus en 2050 contre 12,6 millions en 2005, soit une hausse de $80 \%$ en 45 ans (RoberTBоввÉE 2006). Le nombre de personnes dépendantes augmentera sensiblement, passant de 800000 à 1,2 million en 2040 (DuÉE \& ReBiLlard 2006). D'après l'enquête «Handicaps, Incapacités, Dépendance» (HID) de l'Institut national de la statistique et des études économiques (INSEE) réalisée en 1999,1,2 million de personnes rencontrent des problèmes d'accessibilité à leur logement. Il s'agit majoritairement de personnes âgées (GoILlot \& MoRMiche 2003).

Dans ce contexte, certains promoteurs développent des produits immobiliers à destination des personnes vieillissantes: résidences services, logements adaptés ou même réhabilitations d'anciens logements censés répondre aux besoins nouveaux des résidents. Ces hébergements réalisés en fonction des opportunités foncières prennent peu en compte la qualité de l'environnement géographique.

La question du vieillissement ne constitue d'ailleurs pas un axe structurant des Plans Locaux d'Urbanisme (PLU) et des documents de planification (BorRATA 2008). Or, la prise en compte du vieillissement ne se limite pas à développer des politiques d'accessibilité physique. Les territoires ne sont pas égaux dans leurs capacités à permettre aux résidents âgés d'y demeurer durablement.

Dans de nombreuses études consacrées aux usages de la ville, les vieilles personnes sont souvent occultées, de même que dans les recherches sur les modes de vie (Spellerberg 1996). Des travaux spécifiques aux personnes âgées sont cependant menés depuis une dizaine d'années sur les mobilités résidentielles (LÉvy 1999), la mobilité quotidienne et l'usage des espaces publics. Cependant, ces approches demeurent ciblées. Ainsi, Pochet (1995) se focalise sur les moyens de transport utilisés tandis que Pегхото (1994) travaille sur les espaces publics comme lieux de sociabilité. Du fait de protocoles et d'échelles d'étude différentes, il est difficile de croiser ces résultats. De plus, il paraît nécessaire de hiérarchiser l'ensemble des critères. Plus récemment
LoRD, Joerin \& ThÉRIAUlt (2009) ont proposé une étude complète sur l'évolution des pratiques de mobilité d'un groupe de 102 banlieusards de l'agglomération de Québec entre 1999 et 2006. La typologie de mobilité proposée prend en compte de nombreux critères (établissements et commerces fréquentés, types de transports et éléments qualitatifs sur les individus enquêtés). Basé sur des entretiens qualitatifs, ce travail n'a toutefois pas vocation à être exploitable comme outil opérationnel pour les aménageurs. Il est donc pertinent de développer une approche systémique au moyen d'une méthode intégrant un ensemble de critères, quel que soit le territoire étudié, afin d'obtenir une vision globale des pratiques spatiales et sociales des personnes âgées. Cet outil doit être directement utilisable par les aménageurs et urbanistes.

Le travail proposé s'inscrit dans le cadre d'une réflexion menée avec l'Agence d'Urbanisme de Lyon, la Ville de Lyon, les Universités Lyon 2 (psychologie), Lyon 3 (aménagement) et la Caisse des Dépôts et Consignations. Il a pour objectif de proposer des pistes de réflexion permettant d'adapter les documents d'urbanisme et de planification aux conséquences cumulées du vieillissement de la population et de la gérontocroissance.

Dans cet article, la réflexion portera sur la recherche d'un optimum territorial pour une population de femmes seules, âgées de plus de 80 ans et demeurant en milieu urbain. Une première partie proposera une méthodologie de classification territoriale à partir de la Méthode Hiérarchique Multicritère (MHM) basée sur la mesure relative et les comparaisons par paires (SAATY 1980, 1993). Une seconde partie consistera à étudier objectivement la hiérarchie proposée en analysant la mobilité quotidienne de personnes âgées possédant les mêmes caractéristiques dans deux ensembles résidentiels situés dans un environnement «favorable» et «peu favorable» au moyen de traceurs GPS (Global Positioning System) et de questionnaires. Une troisième partie exposera l'importance de cette classification territoriale pour la population concernée par l'enquête, les enjeux ainsi que les limites opérationnelles pour les pouvoirs publics.

\section{Une classification des territoires}

Le concept d'environnement favorable doit avant tout être défini. Il consiste, au sein d'un territoire donné, 
à dégager des espaces présentant des conditions plus favorables que d'autres par rapport aux besoins et aux pratiques de la population enquêtée. Il s'agit alors de déterminer des indicateurs qui permettront de qualifier l'espace comme étant favorable ou, au contraire, non favorable. Le $8^{\text {ème }}$ arrondissement de Lyon a été choisi comme terrain d'étude car il comprend une très grande hétérogénéité d'habitat (ensemble pavillonnaire, grands ensembles, centres urbains), de transports (bus, métro, tramway), de commerces et de structuration générale (grandes avenues, rues en pentes, impasses).

\subsection{Quels choix pour quels indicateurs?}

Différentes enquêtes montrent que deux éléments sont particulièrement importants: la présence de commerces et l'accessibilité à des moyens de transport adaptés (CENTRE D'ÉTUDES SUR LES RÉSEAUX, LES TRANSPORTS, L'URBANISME ET LES CONSTRUCTIONS PUBLIQUES - CERTU 2004) situés à proximité du lieu de résidence des personnes âgées et facilement accessibles à pied (CERTU 2001), car de nombreuses personnes de 65 ans et plus ne souhaitent pas ou ne peuvent plus conduire (CERTU 2004). Une première tentative de cartographie a été effectuée. Elle comprenait une carte de densité des commerces de proximité (boulangeries, boucheries/charcuteries, tabac/presse, petites et moyennes surfaces, marchés, pharmacies) et des services (banques, bureaux de poste, lieux de culte) préalablement identifiés et géoréférencés. N'étaient sélectionnés que les secteurs comprenant au moins 20 commerces ou services par kilomètre carré car sous ce seuil l'offre des commerces et des services semblait trop disparate et ténue pour l'implantation d'hébergements de personnes âgées. Dans cette première approche, les pentes supérieures à $5 \%$ étaient proscrites mais le choix de ce seuil était largement discutable. Les lignes de bus, tramway et métro (lorsqu'elles sont accessibles pour tous) étaient également cartographiées. Ces cartes de densité permettaient donc de déterminer des zones potentiellement favorables en termes de commerces, services et transports mais devant les difficultés méthodologiques et l'aspect trop théorique de ce travail, une autre approche devait être trouvée. Il était nécessaire d'évaluer et de prendre en compte objectivement les besoins exprimés par la population ciblée.

\subsection{Le choix de la Méthode Hiérarchique Multicritère} Selon Griot (2007: 54), «les méthodes multicritères d'aide à la décision servent à hiérarchiser des éléments et apparaissent donc adaptées pour modéliser la sensibilité». Il est donc fait appel à ces méthodes dans des problèmes de choix ou d'évaluation de situations semblant complexes ou confuses, de manière à faciliter ainsi la prise de décision (BARCZAK \& GRIVAulT 2007). Le but de ces méthodes est l'évaluation de prio- rités entre les différents éléments composant la situation. Il existe plusieurs méthodes d'aide à la décision dont deux qui se distinguent particulièrement: l'école francophone avec des méthodes comme SatisfactionRegret (Fustier 1994) ou ELECTRE (Elimination et choix traduisant la réalité) (Roy 1985), et l'école américaine avec l'Analytic Hierarchy Process (SAATY 1980) ou la Multiattribute Utility Theory (KeEnEY \& RAIFFA 1993).

Ces différentes méthodes ont fait l'objet d'évaluation sur des critères tels que la pertinence, la fiabilité, l'adaptabilité ou la mise en œuvre (GRIOT 2003). La Méthode Hiérarchique Multicritère (MHM) (KeNDRICK \& SAATY 2007) se dégage nettement ici en raison de sa facilité d'utilisation pour différents types d'études (vulnérabilité urbaine, recherche d'optimum territorial) ou encore sa grande souplesse d'utilisation et d'adaptation (GRIOT 2007).

La MHM de SAATy (1980) a ainsi été choisie dans le cadre de cette étude. Les critères ont été sélectionnés afin d'être homogènes les uns avec les autres. Il s'agit d'en comparer trois principaux: la présence de commerces/services, la présence de transports en commun et la présence d'espaces publics (parcs, squares et places publiques). Chaque critère comprend une série de sous-critères. Cette méthode de mesure des éléments se base sur l'opinion de personnes âgées exprimée lors d'entretiens semi-directifs effectués avec un formulaire dans différents marchés du 8ème arrondissement. Il s'agit exclusivement de femmes seules, de 80 ans et plus et qui résident dans le territoire étudié. Les priorités relatives de ces éléments sont délivrées à l'aide d'un calcul matriciel selon le principe des comparaisons deux à deux (pairwise comparison), où il est demandé à la personne interrogée d'évaluer la «priorité relative» entre les deux éléments proposés (Griot 2003). Chaque élément est comparé à tous les autres éléments du même ordre. Afin de valider la cohérence des réponses fournies par chaque personne âgée interrogée, un ratio de cohérence est calculé à partir des valeurs propres des matrices de comparaison. Ce dernier doit être égal ou inférieur à $10 \% .70$ questionnaires ont été remplis et 52 ont pu être exploités.

Un traitement spécifique est nécessaire pour agréger les jugements des personnes âgées interrogées. L'emploi de la moyenne géométrique a été choisi, puis des vecteurs propres aux matrices de jugement ont été calculés afin de déterminer les priorités via le logiciel Expert Choice ${ }^{\circledR}$.

\subsection{Premiers résultats et SIG}

Les résultats obtenus permettent de prioriser les différents critères à l'aide de fonctions. 
Qualité globale $=0,700 \times$ présence de commerces + 0,171 x présence de transports en commun $+0,129$ x présence d'espaces publics de qualité (1).

L'importance des «commerces de proximité» est clairement mise en avant (équation 1) devant «les transports en commun» puis les «espaces publics de qualité».

En étudiant la «qualité des transports» (équation 2) qui constitue le sous-critère de la "présence de transports en commun», les personnes âgées interrogées privilégient le bus $(57,3 \%)$, le tramway $(32,3 \%)$ et enfin le métro $(10,4 \%)$

Qualité des transports $=0,573 \times$ lignes de bus accessibles $+0,171 \mathrm{x}$ lignes de tramway accessibles $+0,104 \mathrm{x}$ lignes de métro accessibles (2).

Les sous-critères de la «qualité des espaces publics» ne sont pas directement exploitables compte tenu du caractère trop hétérogène des éléments comparés. Toutefois, il convient de noter l'intérêt pour la présence de «parcs, squares et jardins publics» $(42 \%)$.

Le Système d'Information Géographique (SIG) est l'application spécifique de la MHM lorsque celle-ci a pour vocation d'établir des priorités dans le domaine de l'aménagement nécessitant de recourir à une visualisation surfacique des interactions entre les éléments en présence. Le SIG permet de hiérarchiser spatialement et thématiquement la situation, mais surtout de concrétiser visuellement les informations quantitatives et numériques obtenues par la MHM. Seule la «qualité territoriale globale» a été prise en compte dans le SIG.

Si les unités de mesure sont dissemblables (quantité absolue, longueur et surface), elles doivent être harmonisées. Un carroyage permet de standardiser le cadre de collecte et la nature hétérogène des données. Un facteur de quantification est défini comme une variable sans dimension s'échelonnant de 0 à 1 . La valeur 0 indique une absence de la cible dans la maille tandis que la valeur 1 signifie que la quantité de cette cible est la plus importante comparativement aux autres mailles. Les facteurs de quantification permettent donc d'obtenir une quantification normalisée de chaque type de cibles.

Ainsi, le facteur de quantification de données surfaciques environnementales (places publiques, squares et parcs) est déterminé par l'équation (3) $: S_{i}=M_{i}$ / $M_{\max }$ où $M_{i}$ représente la superficie de la cible $i$ dans la maille en question et où $\mathrm{M}_{\max }$ représente la superficie maximale de la cible $i$ dans une maille du territoire étudié.
De la même manière, le facteur de quantification de données linéaires environnementales ou matérielles est déterminé par l'équation (4) : $\mathrm{L}_{i}=\mathrm{Q}_{i} / \mathrm{Q}_{\max }$ où $\mathrm{Q}_{i}$ représente la longueur de la cible environnementale ou matérielle $i$ dans la maille en question et où $\mathrm{Q}_{\max }$ représente la longueur maximale de la cible $i$ dans une maille du territoire étudié. Il convient de remarquer que les unités des données de superficie ou de longueur sont libres (le $\mathrm{km}^{2}$ peut aussi bien être employé que le $\mathrm{m}^{2}$ ), car l'établissement des facteurs de quantification repose sur un quotient de valeurs.

Enfin, le facteur de quantification de données ponctuelles (commerces de proximité, arrêts de bus, tramway ou métro) s'exprime par (5): $\mathrm{P}_{i}=\mathrm{R}_{i} / \mathrm{R}_{\text {max }}$ où $\mathrm{R}_{i}$ représente la quantité d'éléments de la cible $i$ dans la maille en question et où $\mathrm{R}_{\max }$ représente la quantité maximale de la cible $i$ dans une maille du territoire étudié.

La représentation spatiale fait ressortir quatre grandes catégories d'environnements: favorable, moyennement favorable, peu favorable et défavorable (Figure 1). Globalement, peu de secteurs sont très favorables excepté le quartier Montplaisir Lumière, au nord de l'arrondissement, qui cumule un nombre important de commerces de proximité, de transports et d'espaces verts. A l'échelle du Plan Local d'Urbanisme (PLU), le niveau de détail pourrait atteindre la parcelle.

Cependant, l'optimum présenté par cette classification pourrait laisser le lecteur perplexe. Les souhaits des personnes âgées interrogées se traduisent-ils concrètement par des actes quotidiens? En effet, la méthode donne uniquement une cartographie subjective des souhaits des personnes interrogées. Par ailleurs, la classification proposée est-elle pertinente au regard des usages de l'espace de proximité par la population concernée? Une étude objective s'avère nécessaire. Pour ce faire, les pratiques spatiales de personnes âgées seules de plus de 80 ans sont alors étudiées dans deux ensembles résidentiels sociaux.

Le premier ensemble, appelé Tony Garnier, est implanté dans un territoire évalué comme «favorable» tandis que le deuxième ensemble, appelé Laennec, se situe dans un environnement jugé «peu favorable» (Figure 1). Afin que les conditions de l'enquête soient optimales, les résidences comportent les mêmes caractéristiques d'équipement. Il y a ainsi des ascenseurs dans toutes les montées concernées par l'étude et les cheminements ne posent pas de problèmes particuliers (absence de marches d'escaliers ou d'obstacles). En effet, il n'aurait pas été pertinent d'étudier des espaces résidentiels dont les caractéristiques d'accessibilité auraient été différentes. 


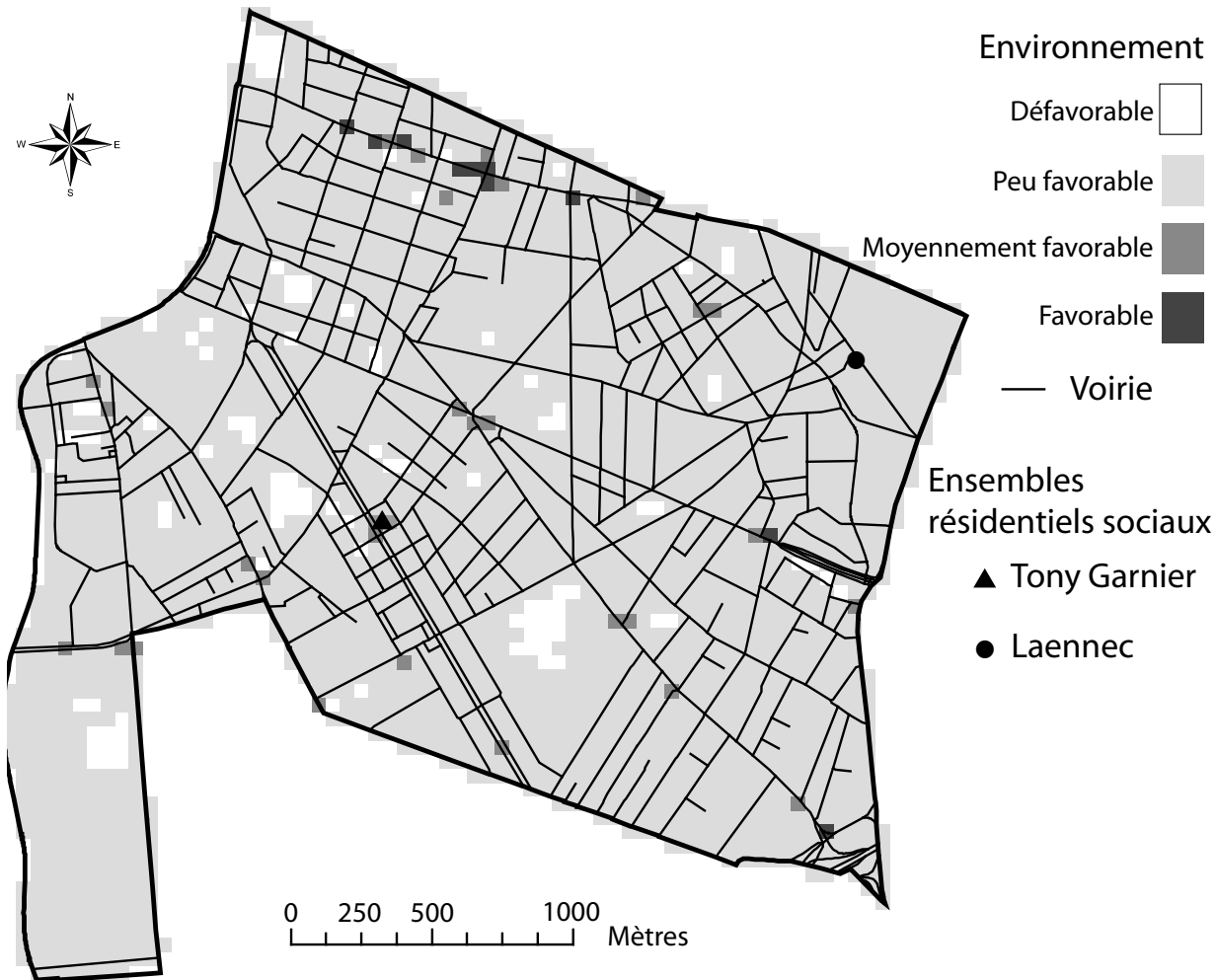

Fig. 1: Représentation spatiale des typologies environnementales du $8^{\text {ème }}$ arrondissement de Lyon en fonction des résultats de la MHM

Spatial representation of environmental typologies of the $8^{\text {th }}$ arrondissement of Lyon determined through a multihierarchical research approach

Räumliche Darstellung der Umwelttypologien im 8. Arrondissement von Lyon auf der Grundlage von Ergebnissen der $M H H$

Source: SIG de l'Université de Lyon III; logiciel: ArcMap 9.1; cartographie: F. Renard et P.-M. ChaPON

\section{Une étude objective des territoires de vie des personnes âgées}

Di Méo (1999) considère qu'il est possible de cartographier l'espace de proximité car il est le fruit de pratiques spatiales répétitives dans leur ensemble, même si le détail des cheminements évolue. Plusieurs méthodes, dont les cartes mentales (LYNCH 1969), permettent de représenter les pratiques spatiales, malgré le fait que ces espaces aient souvent peu de liens avec la réalité car ils sont le fruit d'un filtrage culturel, social et personnel. Une deuxième méthode consiste à utiliser des questionnaires et à effectuer des entretiens qualitatifs. Cependant, le caractère subjectif demeure. Le choix s'est donc porté sur une association de traceurs GPS et de questionnaires.

\subsection{L'utilisation des traceurs GPS}

Le protocole mis en place prévoit d'enquêter auprès de treize résidents dans l'ensemble résidentiel Laennec et de 11 résidents dans l'ensemble Tony Garnier. Des traceurs GPS Tracksticks ${ }^{\circledR}$ autonomes sont ainsi utilisés par les résidents à chaque sortie. Selon les données du constructeur, Active Media Technologie, ces appareils peuvent atteindre une précision de 2,5 mètres en fonction de la qualité de réception des signaux envoyés par les 12 satellites interrogés. Il existe plusieurs travaux scientifiques utilisant les GPS, sur les parcours de vie de personnes souffrant de déficience cognitive (WAHL et al. 2008) ou encore le comportement d'enfants autistes.

Les personnes sélectionnées et volontaires pour 
l'étude sont équipées d'un traceur pour une durée d'une semaine. L'expérimentation s'est déroulée entre fin septembre et fin octobre 2008. Pour compléter les données des traceurs GPS, les personnes volontaires complètent chaque soir un tableau récapitulatif de leurs déplacements dans la journée qui permet de savoir si la personne était seule ou accompagnée lors de ses déplacements, ainsi que le type de transport utilisé (transports en commun, taxi, famille).

Le travail d'analyse se décompose en plusieurs étapes. Les déplacements effectués à pied sont traités séparément des déplacements effectués en transports en commun (métro ou bus). Un premier travail consiste à réaliser des zones tampons de 300 et 500 mètres autour du lieu de résidence dans les deux cas étudiés, afin d'avoir une vision précise des trajets couverts et des distances parcourues. Une cartographie de la densité des lieux ponctuels visités est ensuite réalisée pour les trajets effectués à pied et en transports en commun, dans le but d'identifier et de cerner les pôles attractifs. Enfin, les lieux visités par les personnes âgées sont représentés par des symboles ponctuels de tailles proportionnelles au nombre de visites. Ainsi, on dispose d'informations précises portant sur les trajets linéaires effectués, la distribution spatiale des lieux visités et l'intensité de ces visites.

\subsection{Analyse des zones tampons: la vérification de l'environnement géographique de proximité favorable}

L'enquête combinée des GPS et des questionnaires révèle de nombreux déplacements dont 243 sont directement exploitables car ils correspondent à un déplacement complet entre deux points identifiables au niveau des GPS et/ou des questionnaires. Il s'agit de points pour lesquels le but du déplacement a pu être analysé grâce notamment à des recoupements entre les données brutes des traceurs et les réponses aux questionnaires qui font clairement apparaître les objectifs du déplacement (Tableau 1).

L'ensemble des données est cartographié au moyen de cartes de densité et d'intensité (Figures 2 et 3 ). Les seuils des densités correspondent à la méthode de discrétisation des intervalles réguliers. Il convient de noter que sur l'ensemble des déplacements exploités, 230 sont réalisées par des personnes âgées non accompagnées, soit près de $95 \%$ des trajets.

Les déplacements en transports en commun sont étudiés en fonction des éléments fournis dans les questionnaires par les résidents et permettent de comprendre des ruptures de signal (p.ex. métro, bâtiment). L'analyse des zones tampons des déplacements de piétons pour l'ensemble résidentiel Tony Garnier fait ressortir que la très grande majorité des déplacements

\begin{tabular}{|l|c|c|}
\hline Type de déplacements & Nombre & Pourcentages \\
\hline Boulangerie & 46 & 18,93 \\
Marché & 34 & 13,99 \\
Pharmacie & 27 & 11,11 \\
Presse & 26 & 10,69 \\
Supermarché & 16 & 6,58 \\
Epicerie & 9 & 3,70 \\
Mercerie & 4 & 1,64 \\
Poste & 4 & 1,64 \\
Pressing & 3 & 1,23 \\
Grands magasins & 18 & 7,40 \\
Médecin & 7 & 2,88 \\
Visite famille/amis & 7 & 0,82 \\
Visite proche hôpital & 37,23 \\
Visite Cimetière & 7 & 15,28 \\
\hline Autre identifié & 7 & \\
\hline
\end{tabular}

Tab. 1: Synthèse des principaux déplacements étudiés

Overview of the main movement of residents

Synthese der wichtigsten Bewegungen im Raum

s'effectue dans un rayon de 500 mètres (Figure 2). Ainsi, $75 \%$ des déplacements tous types de transports confondus s'effectuent dans ce rayon. Le territoire de vie s'établit donc à l'échelle du quartier.

L'analyse des déplacements des résidents de Laennec est plus complexe (Figure 3). Une partie des personnes se déplaçant à pied parcourt des distances plus importantes pour se rendre dans les rares commerces de proximité et dépasse les 700 mètres pour atteindre les grands magasins, déplaçant ainsi le centre de densité des lieux fréquentés. En outre, il existe de fortes pentes entre l'ensemble résidentiel et les grands magasins. Les déplacements effectués à pied ne représentent que $40 \%$ des déplacements tous modes confondus dans l'ensemble Laennec contre $91 \%$ des déplacements des résidents de Tony Garnier. 


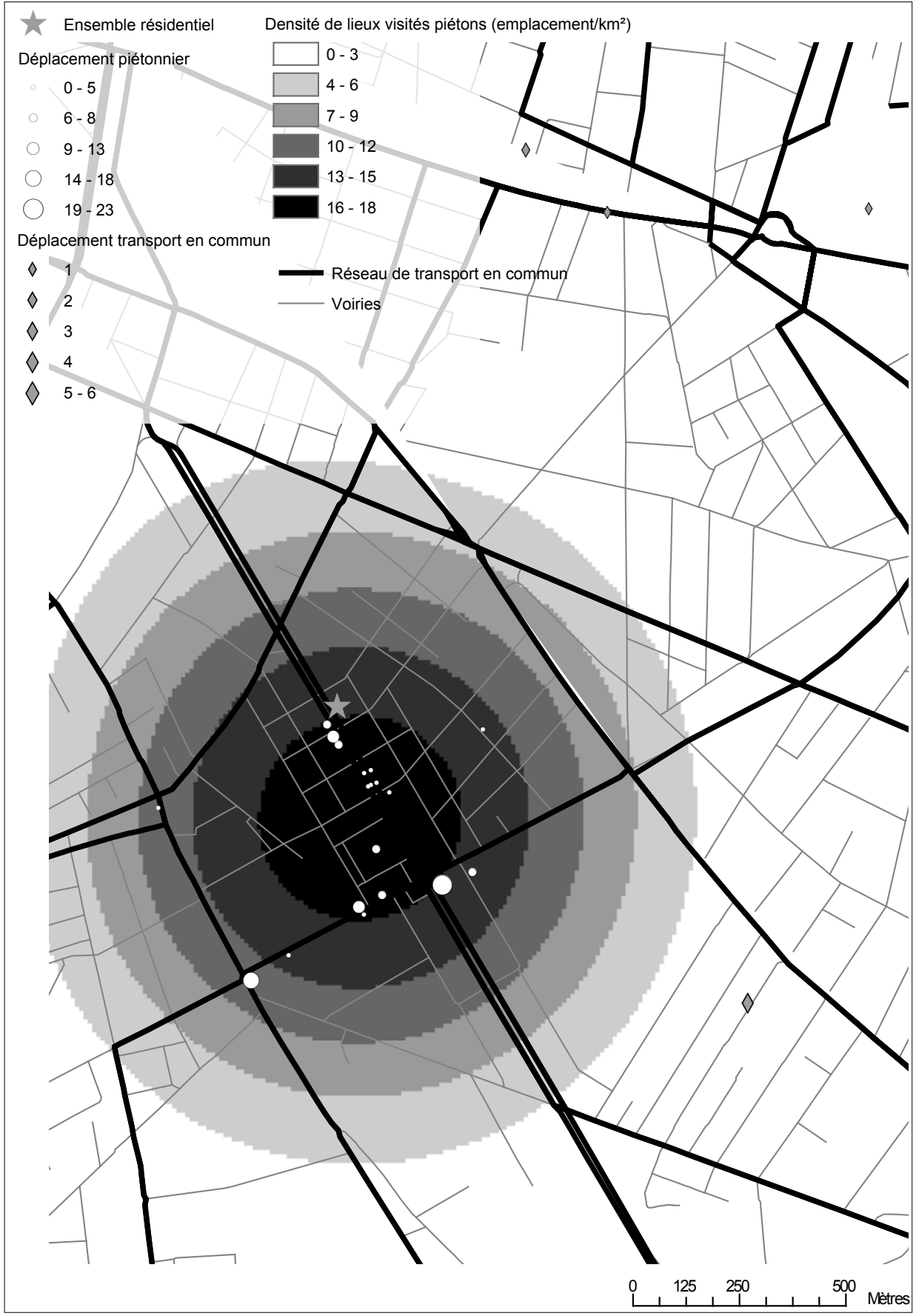

Fig. 2: Densité et intensité des lieux visités par les résidentes de l'ensemble Tony Garnier Density and intensity of the places visited by the Tony Garnier female residents Dichte und Intensität der von Bewohnerinnen von Tony Garnier besuchten Orte Source: SIG de l'Université de Lyon III; logiciel: ArcMap 9.1; cartographie: F. RENARD et P.-M. ChAPON 


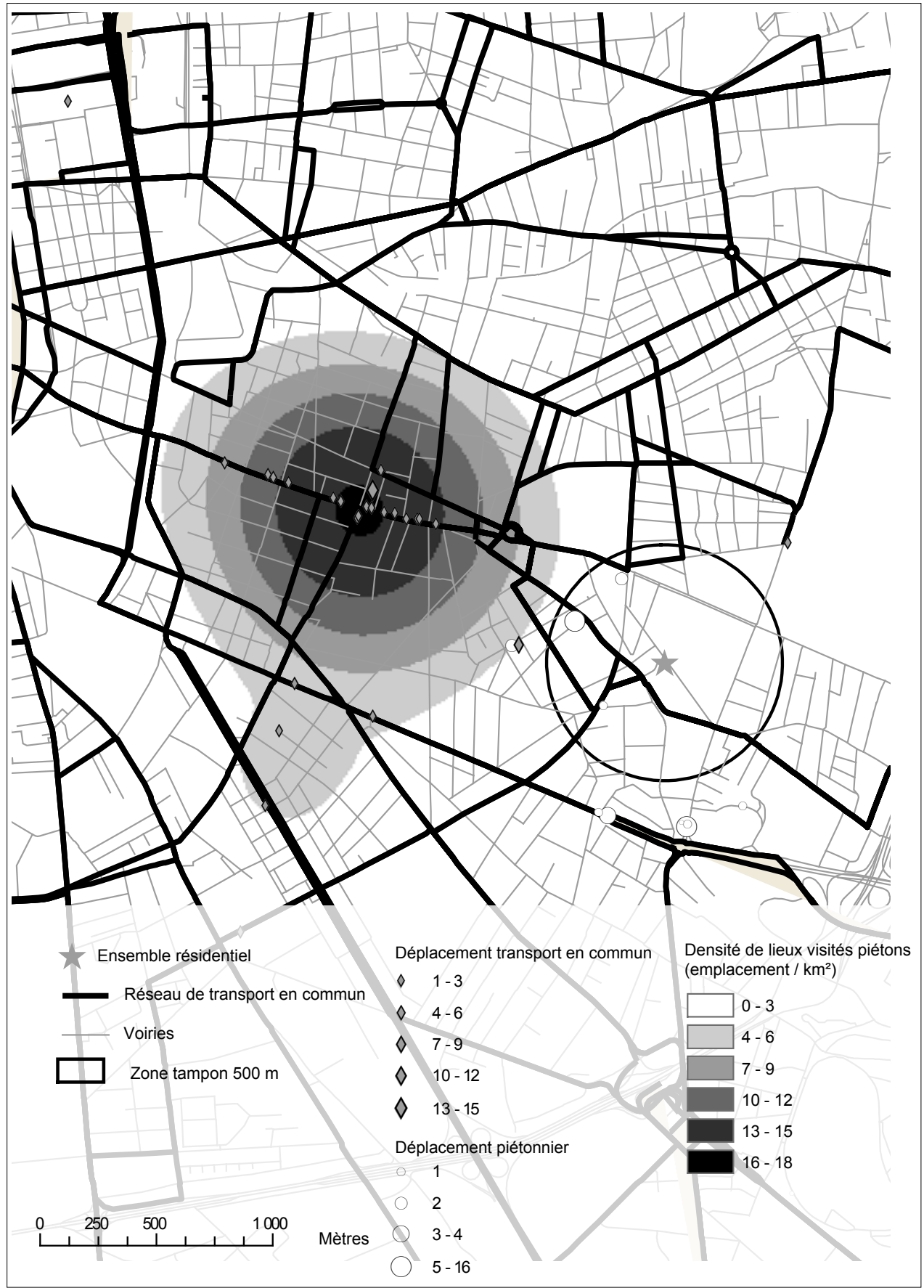

Fig. 3: Densité et intensité des lieux visité par les résidentes de l'ensemble Laennec Density and intensity of the places visited by the Laennec female residents Dichte und Intensität der von Bewohnerinnen von Laennec besuchten Orte Source: SIG de l'Université de Lyon III; logiciel: ArcMap 9.1; cartographie: F. RENARD et P.-M. CHAPON 


\subsection{Des territoires de vie «déplacés» grâce aux transports en commun}

L'analyse de la densité des lieux parcourus par les transports en commun fait ressortir l'importance du quartier commerçant de Montplaisir Lumière (Figure 3). Plus de la moitié des déplacements des habitants de Laennec s'effectue ainsi dans ce territoire dont l'aire de proximité n'excède pas 500 mètres. Les résidents prennent le métro au pied de l'immeuble et se rendent dans ce quartier situé à quelques stations. On retrouve à Montplaisir une aire comparable à celle constatée dans le quartier de Tony Garnier (Figure 2). Ainsi, le métro agit comme un connecteur entre l'ensemble résidentiel Laennec et le quartier Montplaisir qui devient majoritairement le quartier au sens affectif et pratique pour les résidents déplacés.

Le quartier de Montplaisir est un pôle d'attraction également pour les habitants de Tony Garnier, qui y effectuent environ $5 \%$ de l'ensemble de leurs déplacements, particulièrement au marché place Ambroise Courtois.

\section{Territoires adaptés: enjeux et limites}

En étudiant précisément ces déplacements effectués quotidiennement, on constate que $15 \%$ des personnes concernées par l'enquête ont effectué moins de deux sorties dans la semaine pour l'ensemble résidentiel Laennec tandis que tous les résidents de Tony Garnier sont sortis au moins deux fois de leur domicile.

\subsection{Un nécessaire approfondissement pluri- disciplinaire}

Bien entendu, il convient d'analyser ce phénomène plus précisément et il aurait été pertinent d'avoir accès au degré de dépendance des résidents. Toutefois, le choix de l'échantillonnage laisse supposer qu'au-delà d'un certain seuil, les résidents n'ont plus la capacité de prendre les transports en commun et se retrouvent isolés géographiquement, n'ayant pas de commerces et d'activités au pied de leur immeuble. A l'inverse, à échantillon comparable, les résidents sortent plus facilement dans l'ensemble résidentiel Tony Garnier. On comptabilise d'ailleurs 132 déplacements dans cet ensemble résidentiel alors qu'on en compte seulement 111 pour Laennec bien que les enquêtes y soient menées sur 13 résidents au lieu de 11.

Une étude approfondie mesurant notamment l'isolement relationnel (PAN Ké SHON 1999) des individus appartenant à un échantillon plus important mériterait d'être menée avec des psychologues afin de vérifier l'observation réalisée dans le cadre de ce premier travail.

\subsection{La limite des stratégies d'adaptation en milieu hostile}

LORD (2004) a montré dans un travail portant sur un ensemble périurbain québécois que lorsque des personnes âgées vivent dans des territoires hostiles, elles aspirent malgré tout à y vieillir et mettent en œuvre des stratégies d'adaptation. D'ailleurs, une très grande majorité des personnes âgées vivant en banlieue désirent y demeurer, la vie en pavillon offrant même des avantages lorsqu'on devient dépendant, car les maisons sont souvent de plain-pied et plus facilement adaptables qu'un appartement (RENAUD 2000).

Malgré son caractère inadapté du fait de la dépendance à l'automobile, l'attachement au milieu contrebalance les obstacles quotidiens (DESPRÈs \& LORD 2005; WAGNILD 2001). D'ailleurs, en France comme en Suisse, on ne constate pas un fort attrait des villes-centres pour les personnes âgées, excepté pour les 50-74 ans (RÉrAT et al. 2008).

Dès lors qu'ils peuvent se déplacer en véhicule, les banlieusards âgés ont plutôt une bonne qualité de vie (LoRD \& LuXEMbourg 2006). Lorsqu'il ne leur est plus possible d'être véhiculés, les résidents se retrouvent alors isolés. Ces études s'inscrivent en parallèle des présents travaux menés en milieu urbain, l'usage ou l'incapacité d'usage de la voiture remplaçant l'usage ou le non usage des transports en commun.

L'optimum territorial est variable selon les catégories de populations étudiées. Ainsi, une autre enquête qui est menée sur une population résidant en Maisons d'Accueil Rurales pour Personnes Âgées (MARPA) dans le département de l'Ain propose des lieux d'implantation dans des territoires accessibles, à proximité d'un lieu de vie (par exemple un lotissement où vivent des familles) mais pas nécessairement à proximité de commerces et services (CHAPON 2009). D'autres catégories de population privilégieront d'autres critères d'implantation entrainant parfois des conflits entre les générations qui se côtoient au sein d'un même foyer (CAIlly \& Dodier 2007).

\section{Conclusion}

Si la construction de logements à l'architecture adaptée peut avoir pour but de fluidifier l'offre de logements en milieu urbain, l'objectif consiste plutôt à attirer une population plus jeune, correspondant à l'âge de la prise de la retraite (RogERs 1988), et ainsi permettre à cette population d'accéder à ce qu'on appelle une «Haute Qualité du Vieillissement» (HQV) en référence à la «Haute Qualité Environnementale» (HQE) dans une approche durable de la cité. 
L'étude présentée montre l'importance de la qualité territoriale pour la population étudiée. Cette classification des environnements géographiques favorables a été proposée au Ministère du logement en France en février 2009 et a reçu un écho favorable. Des constructions neuves ou des aménagements réalisés dans des périmètres territoriaux établis au moyen de cette approche pourraient entrer dans un cadre fiscal particulier (réduction de la taxe sur la valeur ajoutée (TVA), crédits d'impôts voire utilisation d'un bonus/ malus territorial) pour contrebalancer l'augmentation du coût du foncier.

\section{Bibliographie}

Barczak, A. \& C. Grivault (2007): Geographical information system for the assessment of vulnerability to urban surface runoff. - In: Actes de Novatech, 6e conférence internationale «Techniques et stratégies durables pour la gestion des eaux urbaines par temps de pluie» 25, 26 et 27 juin 2007, Lyon, France, vol. 1, Lyon, édition GRAIE (Groupe de Recherche Rhône-Alpes sur les Infrastructures et l'Eau): 131146.

Borrata, S. (2008): Le vieillissement dans les documents de planification des agglomérations grenobloise, lyonnaise et stéphanoise. - In: AgENCE D'URBANISME POUR LE DÉVELOPPEMENT DE L'AGGLOMÉRATION LYONNAISE (éd.): Le vieillissement, à l'épreuve des territoires et des politiques publiques dans les grandes agglomérations rhônalpines. - Lyon: Agence d'urbanisme pour le développement de l'agglomération lyonnaise.

CAILly, L. \& R. Dodier (2007): La diversité des modes d'habiter des espaces périurbains dans les villes intermédiaires: différenciations sociales, démographiques et de genre - In: Norois 205, 4: 67-80

CENTRE D'Études SUR LES RÉSEAUX, LES TRANSPORTS, L'URBANISME ET LES CONSTRUCTIONS PUBLIQUES (CERTU) (2001): La mobilité des personnes âgées. Analyse des enquêtes ménages déplacements. - Lyon: CERTU.

CENTRE D'ÉTUdes SUR Les RÉSEAUX, LES TRANSPORTS, L'URBANISME ET LES CONSTRUCTIONS PUBLIQUES (CERTU) (2004): Chiffres clés des enquêtes ménages déplacements. - Lyon: CERTU.

Chapon, P.-M. (2009): Quelles implantations pour la création de futures Maisons d'Accueil Rurales pour Personnes Âgées (MARPA)? Etude à partir de cartes mentales. - In: Actes de la conférence «Territoires adaptés au vieillissement et aux modes de vie: enjeux et limites», 25 mars 2009, Lyon, France. - Lyon: Agence d'urbanisme pour le développement de l'agglomération lyonnaise.

DESPRÈs, C. \& S. LoRD (2005): The meaning of home for elderly suburbanites. - In: RowLES, G.D. \& H. Chaudhury (éds): Coming home: International per- spectives on place, time and identity in old age. - New York: Springer: 317-340.

Di MÉo, G. (1999): Géographies tranquilles du quotidien: une analyse de la contribution des sciences sociales et de la géographie à l'étude des pratiques spatiales - In: Cahiers de géographie du Québec 118, 43: 75-93.

DuÉe, M. \& C. Rebillard (2006): La dépendance des personnes âgées: une projection en 2040. - In: Données sociales, Institut national de la statistique et des études économiques - INSEE.

Fustier, B. (1994): Prise de décision sur des critères qualitatifs. - Université de Corse, Centre d'Economie et de Modélisation Appliquées, Note de recherche 18. Goillot, C. \& P. Mormiche (2003): Les enquêtes Handicaps-Incapacités-Dépendance de 1998 et 1999. Résultats détaillés. - In: INSEE (Institut national de la statistique et des études économiques) Résultats, no 22.

Griot, C. (2003): Vulnérabilité et risques liés au transport de matières dangereuses. Evaluation de la vulnérabilité en vue de la préparation aux interventions de la Sécurité Civile. - 2 volumes, Thèse de Doctorat de troisième cycle, Faculté de Géographie, Université Paul Valéry, Montpellier III.

GRIOT, C. (2007): Des territoires vulnérables face au transport de matières dangereuses. Proposition d'un outil d'aide à la gestion de crise. - In: Géocarrefour 82,1/2: 51-63.

Keeney, R.L. \& H. Raiffa (1993): Decisions with multiple objectives-preferences and value tradeoffs. Cambridge et New York: Cambridge University Press. KendRICK, J.D. \& D. SAATY (2007): Use analytic hierarchy process for project selection. - In: Six Sigma Forum Magazine 6, 4: 22-29.

LÉvy, J.-P. (1999): La mobilité résidentielle des plus de 50 ans: l'exemple de la Seine-Saint-Denis. - In: Les cahiers de l'institut d'aménagement et d'urbanisme de la région Île-de France 2, 122.

LoRD, S. (2004): Aging at home in suburbs: A comparison of three age groups with regards to territorial mobility and residential aspirations. - Proceedings of the $18^{\text {th }}$ Conference of the International Association for People-Environment Studies (IAPS), 10-14 juillet 2004, Vienna.

Lord, S. \& N. Luxembourg (2006): The mobility of elderly residents living in suburban and periurban territories: A comparison of residential aspirations and practices in Canada and France. - In: Journal of Housing for the Elderly 4, 20: 103-121.

Lord, S., Joerin, F. \& M. Thériault (2009): Évolution des pratiques de mobilité dans la vieillesse: un suivi longitudinal auprès d'un groupe de banlieusards âgés. - In: Cybergeo, www.cybergeo.eu/index22090.html 23.02.2009.

Lynch, K. (1969): L'image de la cité. - Paris: Dunod. Pan Ké Shon, J-L. (1999): Vivre seul, sentiment de soli- 
tude et isolement relationnel. - In: INSEE Première no 678 , Institut national de la statistique et des études économiques.

Peixoto, C. (1994): Les personnes âgées dans les espaces publics et le désir de plaire. - In: Gérontologie et société 69: 150-159.

Pochet, P. (1995): Mobilité quotidienne des personnes âgées en milieu urbain: évolution récente et perspectives. - Thèse de Doctorat, Faculté des Sciences Economiques de Gestion, Université Lumière, Lyon.

Renaud, F. (2000): Les aîné(e)s et le logement. Un profil quantitatif de la situation résidentielle des aînés au Québec. - Québec: Société d'Habitation du Québec.

Rérat, P., Piguet, E., Besson, R. \& O. Söderström (2008): Les âges de la ville. Mobilité résidentielle, parcours de vie et attractivité des villes suisses. - In: Geographica Helvetica 63, 4: 261-271.

Robert-Bobée, I. (2006): Projections de population pour la France métropolitaine à l'horizon 2050. - In: INSEE Première, no 1089, Institut national de la statistique et des études économiques.

Rogers, A. (1988): Age patterns of elderly migration: an international comparison. - In: Demography 25, 3: 355-377.

Roy, B. (1985): Méthodologie multicritère d'aide à la décision. - Paris: Economica.

SaAty, T.L. (1980): The Analytic Hierarchy Process. - New York: McGraw-Hill.

SAATY, T.L. (1993): What is relative measurement? The ratio scale phantom. - In: Mathematical and Computer Modelling 17, 4/5: 1-12.

SpellerberG, A. (1996): Soziale Differenzierung durch Lebensstile. Eine empirische Untersuchung zur Lebensqualität in West- und Ostdeutschland. - Berlin: Sigma.

WAGNILD, G. (2001): Growing old at home. - In: Journal of Housing for the Elderly 14, 1: 71-84.

Wahl, H.W., Oswald, F., Voss, E., Schilling, O. \& T. FREYTAG (2008): Tracking technology as a means to learn about outdoor mobility in a cognitively impaired older adults. - In: Gerontechnology 7, 2.

\section{Résumé: Construire des logements adaptés aux personnes âgées: une analyse par les territoires de vie à Lyon (France)}

L'objet de cette étude consiste à développer une méthodologie de classification permettant de choisir les emplacements fonciers les plus favorables à la construction de logements adaptés à des femmes seules âgées de plus de 80 ans. La méthode hiérarchique multicritère (MHM) est utilisée et permet de dégager, au sein du $8^{\text {ème }}$ arrondissement de Lyon, des zones optimales pour l'implantation des logements. Toutefois, cette approche subjective doit être complétée par une analyse objective de la mobilité de la population concernée au moyen de traceurs GPS utilisés dans deux ensembles résidentiels comparables du $8^{\text {ème }}$ arrondissement de Lyon. Cet outil permet de faire ressortir les «territoires de vie» de la population concernée et ainsi d'étudier le type, l'intensité et la densité des déplacements. La méthode utilisée dans ce travail pourra ensuite être utilisée dans les documents d'urbanisme et de planification urbaine.

Mots-clés: personnes âgées, hébergements pour personnes âgées, évaluation des territoires, qualité de vie, traceurs GPS

\begin{abstract}
Optimal senior citizen residential planning by means of living space analysis in Lyon, France

The article presents results of a research project to develop a methodology to rank plots of land targeted for development of housing suitable for single women over 80. Use is made of a multi-hierarchical method (MHM) to identify the most appropriate locations in the $8^{\text {th }}$ district of Lyon, France. This subjective approach is complimented by an objective analysis of the mobility of the target population by means of GPS tracking units. For this purpose, residents in two comparable developments in the target district were equipped with these devices. This made it possible to delineate the area within which the senior citizens live and move, and to study the nature, length and frequency of their trips. It is argued that the method implemented in this study is suitable for urban planning and development.
\end{abstract}

Keywords: elderly, senior citizen housing, criteria for urban development schemes, favourable urban environment, high quality of life, GPS trackers

\section{Zusammenfassung: Optimaler seniorengerechter Wohnungsbau durch Analyse von Lebensumfeldern in Lyon (Frankreich)}

Diese Arbeit entwickelt eine Klassifizierungsmethodik für die Wahl bestgeeigneter Grundstücke für den Wohnungsbau von Altenwohnungen für alleinstehende, über achtzigjährige Frauen. Die multi-hierarchische Methode (MHM) wird dazu im 8. Bezirk von Lyon eingesetzt. Allerdings wird dieser subjektive Ansatz durch eine objektive Analyse der Mobilität der Bevölkerung mit Hilfe von GPS-Tracking-Einheiten ergänzt. Solche GPS-Geräte werden von Frauen benutzt, die in zwei vergleichbaren Überbauungen im 8. Bezirk von Lyon leben, und ermöglichen es, deren räumlichen Aktionsradius einzugrenzen, ferner die Art, Dauer und Häufigkeit räumlicher Bewegungen zu untersuchen. Die Methode dieser Studie kann in der Stadtplanung und -entwicklung verwendet werden. 
Schlüsselwörter:Hochbetagte, Altenwohnungen, Wohngebietsbewertung, Lebensqualität, Plotter GPS

Géogr. dipl. Pierre-Marie Chapon, Unité Mixte de Recherche (UMR) 5600 «Environnement, Ville, Société», 18, Rue Chevreul, F-69362 Lyon cedex 07, France et ICADE, 35, Rue de la Gare, F-75019 Paris, France.

e-mail: pierre-marie.chapon@icade.fr

Géogr. dipl. Florent Renard, Unité Mixte de Recherche (UMR) 5600 «Environnement, Ville, Société», 18, Rue Chevreul, F-69362 Lyon cedex 07, France.

e-mail: florent.renard@gmail.com

\section{Manuskripteingang/received/manuscrit entré le} 19.2.2009

Annahme zum Druck/accepted for publication/accepté pour l'impression: 4.9.2009 